\title{
CONTINUOUS MIXTURES OF EXPONENTIALS AND IFR GAMMAS HAVING BATHTUB-SHAPED FAILURE RATES
}

\author{
HENRY W. BLOCK, ${ }^{* * * * * *}$ University of Pittsburgh \\ NAFTALI A. LANGBERG, ${ }^{* * * *}$ University of Haifa \\ THOMAS H. SAVITS *** AND \\ JIE WANG, ${ }^{*}$ University of Pittsburgh
}

\begin{abstract}
It can be seen that a mixture of an exponential distribution and a gamma distribution with increasing failure rate for the right choice of parameters can yield a distribution with a bathtub-shaped failure rate. In this paper we consider a continuous mixture of exponentials and a continuous mixture of gammas with increasing failure rates and show that the resulting mixture has a bathtub-shaped failure rate.
\end{abstract}

Keywords: Reliability; failure rate function; bathtub failure rate

2010 Mathematics Subject Classification: Primary 62N05

\section{Introduction}

We consider mixtures of continuous distributions whose failure rate turns out to have a bathtub failure shape. For extensive discussions concerning mixtures of survival functions and also bathtub-shaped failure rates, see the recent books of Lai and Xie (2006) and also Marshall and Olkin (2007).

Many populations studied in reliability can be considered heterogeneous. The reason for this is that there are usually at least two subpopulations: the normal (sometimes called the strong) subpopulation and the defective (or weak) subpopulation. This implies that the population can be modeled as a mixture of two or more lifetimes. The failure rate function for the population is not just a simple composite of the failure rate of the subpopulations. It is a complicated function whose shape is hard to predict, even if the shape of the failure rates of the subpopulations are very simple.

Several important results about the limiting behavior of failure rates of mixtures of distributions are given in Block et al. (2003). In Example 2.4 therein it was shown that a mixture of an exponential distribution and a gamma distribution with the right choice of parameters can yield a distribution with a bathtub-shaped failure rate. Recently, in Block et al. (2008) the overall behavior of similar mixtures was discussed. In that paper it is shown that when a continuous mixture of exponentials, gammas, or Weibulls with decreasing failure rates are mixed with a gamma with increasing failure rate, under certain conditions on the parameters, a distribution having a bathtub-shaped failure rate is obtained.

Received 21 August 2008; revision received 21 July 2010.

* Postal address: Department of Statistics, University of Pittsburgh, Pittsburgh, PA 15260, USA.

** Supported by the NSA, grant no. H 98230-07-1-0018.

*** Email address: hwb@stat.pitt.edu

**** Postal address: Department of Statistics, University of Haifa, Mount Carmel, Haifa 31999, Israel. 
In the present paper we show that these results can be extended. The single gamma distribution having increasing failure rate, mentioned in the previous paragraph, can be replaced by a continuous mixture of gammas with increasing failure rates and this will still result in a distribution with a nondegenerate bathtub failure rate. Our main result is Theorem 2.1 in Section 2. Details are given in Appendix A.

A few facts about the gamma distribution are needed. The density of the gamma with shape parameter $\gamma>0$ and scale parameter $\lambda>0$ is given by

$$
g(t \mid \lambda, \gamma)=\frac{\lambda^{\gamma}}{\Gamma(\gamma)} t^{\gamma-1} \exp (-\lambda t) \quad \text { for } t>0
$$

and the survival function is denoted by $\bar{G}(t \mid \lambda, \gamma)$. If $\lambda=1$, we use the notation $g(t \mid \gamma)$ and $\bar{G}(t \mid \gamma)$, respectively. We also have, for $t>0$ and $\gamma>2$,

$$
g^{\prime}(t \mid \gamma)=g(t \mid \gamma-1)-g(t \mid \gamma)
$$

and

$$
g^{\prime \prime}(t \mid \gamma)=g(t \mid \gamma-2)-2 g(t \mid \gamma-1)+g(t \mid \gamma) .
$$

We now give the definition of a bathtub distribution.

Definition. A distribution has a bathtub-shaped failure rate if the failure rate function is decreasing up to a point and then increasing after that point. We sometimes use the term bathtub distribution to designate a distribution with a bathtub-shaped failure rate function.

Notes. (a) The terms decreasing and increasing will be used to mean nonincreasing and nondecreasing. If the monotonicity is strict, we will precede the terms with 'strictly'.

(b) A bathtub distribution will be said to be degenerate bathtub if the failure rate function is always increasing or always decreasing. Otherwise it will be said to be nondegenerate bathtub.

\section{Main result}

It was recognized by several authors that distributions with bathtub-shaped failure rates could arise as simple mixtures. For example, Gupta and Warren (2001, Section 3) showed that a certain mixture of two gammas, one with an increasing failure rate (IFR) and the other with a decreasing failure rate (DFR), could have a failure rate which is bathtub. An even simpler example, given in Block et al. (2003, Example 2.4), gives a mixture of an exponential and an IFR gamma which also has a bathtub-shaped failure rate. It turns out that both of these examples are a special case of a much more general result which is given in Block et al. (2008). Mixing an IFR gamma with a host of different DFR distributions turns out to have a failure rate with bathtub shape. In the present paper we show that we can replace the single gamma by a mixture of IFR gammas and obtain the same results. A mixture of IFR gammas is in general not IFR. However, subject to restrictions on the parameters, mixtures of two IFR gammas may be IFR. See Gupta and Warren (2001) where examples and the restrictions on the parameters are given. As part of our main result, we show that our continuous mixture of IFR gammas is also IFR.

In the following theorem we consider a mixture of two quantities: an arbitrary mixture of exponentials and an arbitrary mixture of IFR gammas. Parameters are chosen so that the failure 
rates of these two quantities do not cross and also so that the mixture of IFR gammas turns out to be IFR (see Theorem 2.1(ii)).

Notation. In the following theorem we consider mixtures of exponential distributions with scale mixture random variable $\Lambda$ and probability measure $R$. We also consider mixtures of gamma distributions with shape parameter random variable $\Phi$ and scale parameter $\lambda_{0}>0$. Restrictions for the random variables and parameters for the general case of Theorem 2.1(iii) are as follows.

(A) For the random variable $\Lambda$, we assume that its probability measure has support on a subset of $\left(\lambda_{0}, \infty\right)$.

(B) For the random variable $\Phi$, we assume that it takes nonzero values only in the interval $(\alpha, \beta)$ with $2<\alpha<\beta<\delta(\alpha)$, where $\delta(\alpha)=\min (2 \alpha-1,4 \alpha-6, \alpha+0.5+$ $0.5 \sqrt{8 \alpha-7})$. Also, $\delta(\alpha)$ can be written as

$$
\delta(\alpha)= \begin{cases}4 \alpha-6 & \text { if } 2<\alpha<2.5 \\ 2 \alpha-1 & \text { if } 2.5 \leq \alpha<4 \\ \alpha+0.5+0.5 \sqrt{8 \alpha-7} & \text { if } 4 \leq \alpha\end{cases}
$$

Theorem 2.1. Consider a mixture of the following two families of mixtures:

- an arbitrary mixture of exponentials with density $h_{1}(t)=\mathrm{E}[\Lambda \exp (-\Lambda t)]$, where $\Lambda$ is the mixture random variable with probability distribution $R$; and

- an arbitrary mixture of gammas each with increasing failure rate and the mixture having density

$$
h_{2}(t)=\mathrm{E}\left[\frac{\lambda_{0}{ }^{\Phi} t^{\Phi-1} \exp \left(-\lambda_{0} t\right)}{\Gamma(\Phi)}\right], \quad \lambda_{0}>0,
$$

where $\Phi \geq 1$ is the mixing random variable.

The overall mixture has density

$$
f(t)=p_{1} h_{1}(t)+p_{2} h_{2}(t),
$$

where $0 \leq p_{i} \leq 1, i=1,2$, and $p_{1}+p_{2}=1$. Then,

(i) for $p_{1}=1, f(t)=h_{1}(t)$ has a decreasing failure rate;

(ii) for $p_{1}=0, f(t)=h_{2}(t)$ has an increasing failure rate if $\Phi$ takes values in $[\alpha, \alpha+0.5+$ $0.5 \sqrt{8 \alpha-7}]$, where $\alpha>2$;

(iii) for $0<p_{1}<1$, under the parameter restrictions given in (A) and (B), the mixture has a bathtub-shaped failure rate.

If $R$ has a finite first moment then the mixture is nondegenerate bathtub.

Before giving the proof of Theorem 2.1 we give some examples showing that if the conditions of Theorem 2.1(iii) are not met, we do not obtain the bathtub shape. We also give some notes on the relation to previous results.

The choice of parameters in Theorem 2.1(iii) gives sufficient conditions to guarantee that the mixture is bathtub. It turns out these bounds are not so far away from necessary conditions, as the following examples show. We give four examples where the parameters chosen are very close 
to but not included in the region given in Theorem 2.1(iii). The behaviors of the failure rates for these examples are quite different from the bathtub shape obtained in Theorem 2.1. In fact, there are many additional changes of monotonicity (bathtub has one change from decreasing to increasing).

For all four examples below, we consider a mixture of the form

$$
f(t)=p_{1} \lambda \exp (-\lambda t)+p_{2} g\left(t \mid \alpha_{1}\right)+p_{3} g\left(t \mid \alpha_{2}\right),
$$

where the $g(t \mid \alpha)$ are gamma densities.

Example 2.1. Let $p_{1}=p_{2}=p_{3}=\frac{1}{3}, \lambda=2, \alpha_{1}=1.5$, and $\alpha_{2}=\frac{13}{6}$. The resulting failure rate has a modified bathtub shape (i.e., increasing, decreasing then increasing). Here $1<\alpha_{1}<2<\alpha_{2}$.

Example 2.2. Let $p_{1}=0.05, p_{2}=0.475, p_{3}=0.475, \lambda=1.4, \alpha_{1}=2.1$, and $\alpha_{2}=3$. The failure rate has at least four changes of monotonicity. Here $2<\alpha_{1}<2.5$, but $\alpha_{2}>$ $4 \alpha_{1}-6=2.4$.

Example 2.3. Let $p_{i}$ and $\lambda$ be as in Example 2.2, $\alpha_{1}=2.6$, and $\alpha_{2}=4.3$. The failure rate has at least four changes of monotonicity. In this case, $2.5<\alpha_{1}<4$ and $\alpha_{2}>2 \alpha_{1}-1=4.2$.

Example 2.4. Let $p_{i}$ and $\lambda$ be as in Example 2.2, $\alpha_{1}=4.2$, and $\alpha_{2}=7.5$. The failure rate has at least seven changes of monotonicity. Here $4<\alpha_{1}$ and $\alpha_{2}>\alpha_{1}+0.5+0.5 \sqrt{8 \alpha_{1}-7}=7.28$.

Notes. (a) In regards to Theorem 2.1(ii), Gupta and Warren (2001) considered the mixture of two gammas with shape parameters $a_{1}$ and $a_{2}$ and the same scale parameter. For the quadratic, which is the numerator of $\eta^{\prime}(t)$ given on page 1908 of their paper, it turns out the mixture is IFR if the discriminant is negative. This reduces to $a_{1}>1, a_{2}>1$, and $a_{1}-1>\left(a_{1}-a_{2}+1\right)^{2} / 4$ (this includes Cases 5 and 6 in the paper along with a symmetric version). This yields a slightly larger region than the one given in Theorem 2.1(ii) when $\Phi$ reduces to the corresponding two point mixture distribution. However, it is important to note that the present result is for arbitrary mixtures of IFR gammas.

(b) In particular, we illustrate the Theorem 2.1(ii) result for the mixture of $n$ gammas. Note that, for the mixture of three gammas, the techniques of Gupta and Warren (2001) do not appear to apply. Let $\lambda_{0}=1$ and $a_{1}<a_{2}<\cdots<a_{n}$, and assume that $a_{1}>1$ and $a_{n}<a_{1}+0.5+0.5 \sqrt{8 a_{1}-7}$. By taking $\alpha=a_{1}$, it follows from Theorem 2.1(ii) that the mixture

$$
\sum_{j=1}^{n} p_{j} \exp (-t) \frac{t^{a_{j}-1}}{\Gamma\left(a_{j}\right)}
$$

has an increasing failure rate.

(c) As in Block et al. (2008), we can replace a continuous mixture of exponentials with a continuous mixture of DFR gammas, 'modified' DFR Weibulls, and any survival distribution which is completely monotone.

(d) Theorem 2.1(iii) gives conditions under which the mixture has an increasing failure rate. A paper by Lynch (1999) also gave conditions under which a mixture has increasing failure rate. The present theorem has no conditions other than on the support of the mixing measure, while Lynch assumed the mixing distribution was IFR. 


\subsection{Proof of Theorem 2.1}

For $p_{1}=1$, the result of the theorem follows since mixtures of DFR distributions are DFR. The proof of the result for $p_{1}=0$, which we include at the end of this section, follows from a special case of the proof of the main result. We prove the main result for $\lambda_{0}=1$. The more general case follows from rescaling. For $\lambda_{0}=1$, we have

$$
f(t)=p_{1} \mathrm{E}[\Lambda \exp (-\Lambda t)]+p_{2} \mathrm{E}\left[\frac{\exp (-t) t^{\Phi-1}}{\Gamma(\Phi)}\right],
$$

where $0<p_{1}<1$, the support of the probability $R$ is a subset of $(1, \infty)$, and $\Phi$ is as given in Theorem 2.1(iii). To show that the failure rate function $r(t)=f(t) / \bar{F}(t)$ is bathtub, we examine the function $\eta(t)=-f^{\prime}(t) / f(t)$. If we can show that $\eta^{\prime}(t)$ has one change of sign from negative to positive (i.e. $\eta(t)$ has a nondegenerate bathtub shape) then, by a result of Glaser (1980), $r(t)$ is either IFR or has a nondegenerate bathtub shape.

To prove Theorem 2.1(iii), we need a proposition. This is proven in Appendix A along with several other results that are needed. First, we note that the derivative of $\eta(t)$ is given by

$$
\eta^{\prime}(t)=\frac{-f(t) f^{\prime \prime}(t)+\left(f^{\prime}(t)\right)^{2}}{f^{2}(t)},
$$

and, thus, the signs of $\eta^{\prime}(t)$ and the numerator above are equivalent. We set

$$
k(t)=\left[f^{\prime}(t)\right]^{2}-f(t) f^{\prime \prime}(t) .
$$

Then it is easy to check that

$$
k(t)=p_{1}^{2} A_{11}(t)+p_{2}^{2} A_{22}(t)+p_{1} p_{2} A_{12}(t),
$$

where

$$
A_{k k}(t)=\left[h_{k}^{\prime}(t)\right]^{2}-h_{k}^{\prime \prime}(t) h_{k}(t), \quad k=1,2,
$$

and

$$
A_{12}=2 h_{1}^{\prime}(t) h_{2}^{\prime}(t)-h_{1}^{\prime \prime}(t) h_{2}(t)-h_{2}^{\prime \prime}(t) h_{1}(t) .
$$

We now let $W(t)=\exp (2 t) t^{4-2 \alpha} k(t)$ for $t>0$. Since $\exp (2 t) t^{4-2 \alpha}>0$, the sign change properties of $k(t)$ are the same as the sign change properties of $W(t)$. We now state the proposition.

Proposition 2.1. (i) $\lim _{t \rightarrow 0^{+}} W(t)=-\infty$.

(ii) $\lim _{t \rightarrow \infty} W(t)=\infty$.

(iii) $W(t)$ strictly increases on $(0, \infty)$.

Proof. See Appendix A.

Then $W(t)$ changes sign exactly once from negative to positive and so $\eta(t)$ is nondegenerate bathtub shaped. Thus, from Glaser (1980), $r(t)$ is IFR or nondegenerate bathtub. We can rule out IFR by Lemma A. 2 in Appendix A when $\Lambda$ has a finite mean.

We conclude with the $p_{1}=0$ case. From the proof of Lemma A.1(ii) in Appendix A,

$$
k(t)=\frac{1}{2} \exp (-2 t) \mathrm{E}\left[t^{\Phi_{1}+\Phi_{2}-4} \Delta\left(\Phi_{1}, \Phi_{2}\right)\right],
$$

where $\Phi_{1}$ and $\Phi_{2}$ are independent versions of $\Phi$. Now if $\Phi$ takes values in $[\alpha, \beta]$ then $\left(\Phi_{1}, \Phi_{2}\right)$ 
take values in $[\alpha, \beta] \times[\alpha, \beta]$. It is not hard to see, from the argument at the beginning of Appendix $\mathrm{A}$, that the largest square such that $\Delta\left(a_{1}, a_{2}\right) \geq 0$ for $\left(a_{1}, a_{2}\right) \epsilon[\alpha, \beta] \times[\alpha, \beta]$ has $\alpha>1$ and $\beta=\alpha+0.5+0.5 \sqrt{8 \alpha-7}$. Thus, from Glaser (1980), $f(t)$ has an increasing failure rate.

\section{Appendix A}

We use the following notation:

$$
\begin{aligned}
\Delta\left(a_{1}, a_{2}\right) & =\frac{\left(a_{1}-1\right)\left(a_{2}-a_{1}+1\right)+\left(a_{2}-1\right)\left(a_{1}-a_{2}+1\right)}{\Gamma\left(a_{1}\right) \Gamma\left(a_{2}\right)} \\
& =\frac{\left(a_{1}+a_{2}-2\right)-\left(a_{1}-a_{2}\right)^{2}}{\Gamma\left(a_{1}\right) \Gamma\left(a_{2}\right)} .
\end{aligned}
$$

This quantity is nonnegative if the numerator is nonnegative, which occurs if

$$
a_{1}+0.5-0.5 \sqrt{8 a_{1}-7} \leq a_{2} \leq a_{1}+0.5+0.5 \sqrt{8 a_{1}-7}
$$

for $a_{1} \geq \frac{7}{8}$.

Furthermore, let

$$
Q(\Lambda, \Phi, t)=\frac{(\Lambda-1)^{2} t^{3-\Phi}+2(\Lambda-1)(\Phi-1) t^{2-\Phi}+(\Phi-1)(\Phi-2) t^{1-\Phi}}{\Gamma(\Phi)} .
$$

It is easy to see that $Q(\Lambda, \Phi, t)>0$ by the restrictions on $\Lambda$ and $\Phi$.

We need the following result to prove Proposition 2.1.

Lemma A.1. For the quantities $A_{i j}$ given in (2.1) and (2.2), and, for $t>0$,

(i) $A_{11}(t)=-\frac{1}{2} \mathrm{E}\left[\Lambda_{1} \Lambda_{2}\left(\Lambda_{2}-\Lambda_{1}\right)^{2} \exp \left(-\left(\Lambda_{1}+\Lambda_{2}\right) t\right)\right]$;

(ii) $A_{22}(t)=\frac{1}{2} \exp (-2 t) \mathrm{E}\left[t^{\Phi_{1}+\Phi_{2}-4} \Delta\left(\Phi_{1}, \Phi_{2}\right)\right]$;

(iii) $A_{12}(t)=-\mathrm{E}\left[t^{2 \Phi-4} \Lambda Q(\Lambda, \Phi, t) \exp (-(\Lambda+1) t)\right]$,

where $\Lambda_{1}$ and $\Lambda_{2}$ are independent versions of $\Lambda$, and similarly for $\Phi_{i}, i=1,2$.

Proof. (i) From (2.1),

$$
\begin{aligned}
A_{11}(t) & =\left[h_{1}^{\prime}(t)\right]^{2}-h_{1}(t) h_{1}^{\prime \prime}(t) \\
& =\left(\mathrm{E}\left[\Lambda^{2} \exp (-\Lambda t)\right]\right)^{2}-\mathrm{E}\left[\Lambda^{3} \exp (-\Lambda t)\right] \mathrm{E}[\Lambda \exp (-\Lambda t)] .
\end{aligned}
$$

Hence,

$$
\begin{aligned}
A_{11}(t) & =\mathrm{E}\left[\Lambda_{1}^{2} \Lambda_{2}^{2} \exp \left(-\left(\Lambda_{1}+\Lambda_{2}\right) t\right)-\Lambda_{1}^{3} \Lambda_{2} \exp \left(-\left(\Lambda_{1}+\Lambda_{2}\right) t\right)\right] \\
& =\mathrm{E}\left[\Lambda_{1}^{2} \Lambda_{2}\left(\Lambda_{2}-\Lambda_{1}\right) \exp \left(-\left(\Lambda_{1}+\Lambda_{2}\right) t\right)\right] .
\end{aligned}
$$

By symmetry,

$$
A_{11}(t)=\mathrm{E}\left[\Lambda_{2}^{2} \Lambda_{1}\left(\Lambda_{1}-\Lambda_{2}\right) \exp \left(-\left(\Lambda_{1}+\Lambda_{2}\right) t\right)\right]
$$

Combining these quantities gives

$$
\begin{aligned}
A_{11}(t) & =\frac{1}{2} \mathrm{E}\left[\left(\Lambda_{1}^{2} \Lambda_{2}\left(\Lambda_{2}-\Lambda_{1}\right)^{2}+\Lambda_{2}^{2} \Lambda_{1}\left(\Lambda_{1}-\Lambda_{2}\right)\right) \exp \left(-\left(\Lambda_{1}+\Lambda_{2}\right) t\right)\right] \\
& =-\frac{1}{2} \mathrm{E}\left[\Lambda_{1} \Lambda_{2}\left(\Lambda_{1}-\Lambda_{2}\right)^{2} \exp \left(-\left(\Lambda_{1}+\Lambda_{2}\right) t\right)\right] .
\end{aligned}
$$


(ii) Similarly, from (2.1),

$$
\begin{aligned}
A_{22}(t)= & {\left[h_{2}^{\prime}(t)\right]^{2}-h_{2}(t) h_{2}^{\prime \prime}(t) } \\
= & {[\mathrm{E} g(t \mid \Phi-1)-\mathrm{E} g(t \mid \Phi)]^{2} } \\
& -\mathrm{E} g(t \mid \Phi)[\mathrm{E} g(t \mid \Phi-2)-2 \mathrm{E} g(t \mid \Phi-1)+\mathrm{E} g(t \mid \Phi)] \\
= & {[\mathrm{E} g(t \mid \Phi-1)]^{2}-\mathrm{E} g(t \mid \Phi) \mathrm{E} g(t \mid \Phi-2) . }
\end{aligned}
$$

The above quantity thus becomes

$$
\mathrm{E}\left[g\left(t \mid \Phi_{1}-1\right) g\left(t \mid \Phi_{2}-1\right)-g\left(t \mid \Phi_{1}\right) g\left(t \mid \Phi_{2}-2\right)\right] .
$$

By symmetry, the above quantity can also be written as

$$
\mathrm{E}\left[g\left(t \mid \Phi_{2}-1\right) g\left(t \mid \Phi_{1}-1\right)-g\left(t \mid \Phi_{2}\right) g\left(t \mid \Phi_{1}-2\right)\right] .
$$

Consequently,

$$
\begin{aligned}
A_{22}(t)= & \frac{1}{2}\left(\mathrm{E}\left[g\left(t \mid \Phi_{1}-1\right) g\left(t \mid \Phi_{2}-1\right)-g\left(t \mid \Phi_{1}\right) g\left(t \mid \Phi_{2}-2\right)\right]\right. \\
& \left.+\mathrm{E}\left[g\left(t \mid \Phi_{2}-1\right) g\left(t \mid \Phi_{1}-1\right)-g\left(t \mid \Phi_{2}\right) g\left(t \mid \Phi_{1}-2\right)\right]\right) \\
= & \frac{1}{2} \mathrm{e}^{-2 t} \mathrm{E}\left[t ^ { \Phi _ { 1 } + \Phi _ { 2 } - 4 } \left\{\frac{1}{\Gamma\left(\Phi_{1}-1\right) \Gamma\left(\Phi_{2}-1\right)}-\frac{1}{\Gamma\left(\Phi_{1}\right) \Gamma\left(\Phi_{2}-2\right)}\right.\right. \\
& \left.\left.-\frac{1}{\Gamma\left(\Phi_{2}\right) \Gamma\left(\Phi_{1}-2\right)}\right\}\right]
\end{aligned}
$$

(iii) From (2.2),

$$
A_{12}(t)=2 h_{1}^{\prime}(t) h_{2}^{\prime}(t)-h_{1}(t) h_{2}^{\prime \prime}(t)-h_{1}^{\prime \prime}(t) h_{2}(t) .
$$

Recalling that $g(t \mid \lambda, \gamma)$ is the density of a gamma distribution with scale parameter $\lambda>0$ and shape parameter $\gamma>0$, we have

$$
\begin{aligned}
A_{12}(t)= & -2 \mathrm{E}\left[\Lambda^{2} \exp (-\Lambda t)(g(t \mid 1, \Phi-1)-g(t \mid 1, \Phi))\right]-\mathrm{E}\left[\Lambda^{3} \exp (-\Lambda t) g(t \mid 1, \Phi)\right] \\
& -\mathrm{E}[\Lambda \exp (-\Lambda t)\{g(t \mid 1, \Phi-2)-2 g(t \mid 1, \Phi-1)+g(t \mid 1, \Phi)\}] \\
= & -\mathrm{E}\left[\Lambda \exp (-(\Lambda+1) t) t^{2 \Phi-4} Q(\Lambda, \Phi, t)\right] .
\end{aligned}
$$

We can now prove Proposition 2.1.

Proof of Proposition 2.1. We write

$$
W(t)=p_{1}^{2} W_{11}(t)+p_{2}^{2} W_{22}(t)+p_{1} p_{2} W_{12}(t),
$$


where

$$
W_{j k}(t)=\exp (2 t) t^{4-2 \alpha} A_{j k}(t) \quad \text { for }(j, k) \in\{(1,1),(2,2),(1,2)\} .
$$

Consequently,

$$
\begin{aligned}
& W_{11}(t)=-\frac{1}{2} t^{4-2 \alpha} \mathrm{E}\left[\Lambda_{1} \Lambda_{2}\left(\Lambda_{2}-\Lambda_{1}\right)^{2} \exp \left(-\left(\Lambda_{1}+\Lambda_{2}-2\right) t\right)\right], \\
& W_{22}(t)=\frac{1}{2} \mathrm{E}\left[t^{\Phi_{1}+\Phi_{2}-2 \alpha} \Delta\left(\Phi_{1}, \Phi_{2}\right)\right], \\
& W_{12}(t)=-\mathrm{E}\left[t^{2(\Phi-\alpha)} \Lambda Q(\Lambda, \Phi, t) \exp (-(\Lambda-1) t)\right] .
\end{aligned}
$$

(i) If $\Lambda$ is not degenerate, it is easy to check, as $t \rightarrow 0+$, with $\alpha>2$ and $\Lambda>1$, that $W_{11}(t) \rightarrow-\infty$. Similarly, using the fact that $\Phi>\alpha>2, W_{22}(t) \rightarrow 0$ as $t \rightarrow 0+$. If $\Lambda \geq 1$ and $\Phi \geq 2$, then $W_{12}(t) \leq 0$ for all $t$. Thus, if $R$ is not degenerate then $W(0+)=-\infty$. If $\Lambda$ is degenerate then $W_{11}(t)=0$. But then if $\beta<2 \alpha-1$, we have $W_{12}(0+)=-\infty$, in which case we still have $W(0+)=-\infty$.

(ii) Similarly, as $t \rightarrow \infty, W_{11}(t) \rightarrow 0, W_{22}(t) \rightarrow \infty$, and $W_{12}(t) \rightarrow 0$. In the latter case, we make use of the assumption that the support of $R$ is contained in $(1, \infty)$. Thus, $W(t) \rightarrow \infty$. let

(iii) It is easy to see that $W_{11}(t)$ and $W_{22}(t)$ are increasing. To show that $W_{12}(t)$ is increasing,

$$
\begin{gathered}
g(t)=\Gamma(\Phi)\left(t^{2(\Phi-\alpha)} Q(\Lambda, \Phi, t) \exp (-(\Lambda-1) t)\right) \\
=\exp (-(\Lambda-1) t)\left[(\Lambda-1)^{2} t^{\Phi-2 \alpha+3}+2(\Lambda-1)(\Phi-1) t^{\Phi-2 \alpha+2}\right. \\
\left.+(\Phi-1)(\Phi-2) t^{\Phi-2 \alpha+1}\right] .
\end{gathered}
$$

Then

$$
\begin{aligned}
g^{\prime}(t)=\exp (-(\Lambda-1) t)[ & -(\Lambda-1)^{3} t^{\Phi-2 \alpha+3}+(\Lambda-1)^{2}(-\Phi-2 \alpha+5) t^{\Phi-2 \alpha+2} \\
+ & (\Lambda-1)(\Phi-1)(\Phi-4 \alpha+6) t^{\Phi-2 \alpha+1} \\
+ & \left.(\Phi-1)(\Phi-2)(\Phi-2 \alpha+1) t^{\Phi-2 \alpha}\right] .
\end{aligned}
$$

We now show the four coefficients above are negative. This is obvious for the first since $\Lambda>1$. For the second coefficient, since $\Phi>\alpha>2$, it follows that $-\Phi-2 \alpha+5<-3 \alpha+5<$ $-1<0$. For the third coefficient, we require that $\Phi-4 \alpha+6<0$, and, for the last coefficient, we need $\Phi-2 \alpha+1<0$. These conditions become $\beta<4 \alpha-6$ and $\beta<2 \alpha-1$, respectively, which we have assumed. This means that $g(t)$ is decreasing and so $W_{12}(t)$ is increasing.

We need one last lemma.

Lemma A.2. The mixture in Theorem 2.1 has a nondegenerate bathtub shape if $R$ has a finite first moment.

Proof. First, note that

$$
r^{\prime}(t)=\frac{f^{\prime}(t) \bar{F}(t)+f^{2}(t)}{\bar{F}^{2}(t)} \quad \text { for } t>0
$$

and so, for $0<p_{1}<1$,

$$
\begin{aligned}
\lim _{t \rightarrow 0+} r^{\prime}(t) & =f^{\prime}(0)+f^{2}(0) \\
& =p_{1}^{2}(\mathrm{E}[\Lambda])^{2}-p_{1} \mathrm{E}\left[\Lambda^{2}\right] \\
& =-p_{1} \operatorname{var} \Lambda-p_{1}\left(1-p_{1}\right) \mathrm{E}^{2} V \\
& <0 .
\end{aligned}
$$

Consequently, $r(t)$ cannot be IFR and so must have a degenerate bathtub shape. 


\section{Acknowledgement}

We would like to thank the anonymous referee for helping to streamline this paper and, consequently, improving the readability.

\section{References}

Block, H. W., LI, Y. AND SAVITS, T. H. (2003). Initial and final behaviour of failure rate functions for mixtures and systems. J. Appl. Prob. 40, 721-740.

Block, H. W., LI, Y., SAvits, T. H. AND WANG, J. (2008). Continuous mixtures with bathtub-shaped failure rates. J. Appl. Prob. 45, 260-270.

Glaser, R. E. (1980). Bathtub and related failure rate characterizations. J. Amer. Statist. Assoc. 75, 667-672.

Gupta, R. C. AND WarRen, R. (2001). Determination of change points of non-monotonic failure rates. Commun. Statist. Theory Meth. 30, 1903-1920.

LaI, C.-D. And XIE, M. (2006). Stochastic Ageing and Dependence for Reliability. Springer, New York.

LYNCH, J. D. (1999). On conditions for mixtures of increasing failure rate distributions to have an increasing failure rate. Prob. Eng. Inf. Sci. 13, 33-36.

Marshall, A. W. ANd OlKIn, I. (2007). Life Distributions. Springer, New York. 